\title{
Screening a mushroom extract library for activity against Acinetobacter baumannii and Burkholderia cepacia and the identification of a compound with anti-Burkholderia activity
}

William R Schwan ${ }^{1 *}$, Craig Dunek ${ }^{1,2}$, Michael Gebhardt ${ }^{1}$, Kathleen Engelbrecht ${ }^{1}$, Tiffany Klett ${ }^{1}$, Aaron Monte ${ }^{2}$, Joseph Toce', Marc Rott', Thomas J Volk², John J LiPuma ${ }^{4}$, Xue-Ting Liu², Ronald McKelvey²

\begin{abstract}
Background: Acinetobacter baumannii and species within the Burkholderia cepacia complex (BCC) are significant opportunistic bacterial pathogens of humans. These species exhibit a high degree of antibiotic resistance, and some clinical isolates are resistant to all currently available antimicrobial drugs used for treatment. Thus, new drugs are needed to treat infections by these species. Mushrooms could be a potential source for new drugs to treat $A$. baumannii and BCC infections.

Methods: The aim of this study was to screen a library of crude extracts from 330 wild mushrooms by disk diffusion assays for antibacterial activity against A. baumannii and Burkholderia cepacia in the hope of identifying a novel natural drug that could be used to treat infections caused by these species. Once positive hits were identified, the extracts were subjected to bioassay-guided separations to isolate and identify the active drug molecules. MICs were performed to gauge the in vitro activity of the purified compounds.
\end{abstract}

Results: Only three crude extracts $(0.9 \%)$ had activity against A. baumannii and B. cepacia. Compounds from two of these extracts had MICs greater than $128 \mu \mathrm{g} / \mathrm{ml}$, and further analyses were not performed. From the third extract, prepared from Leucopaxillus albissimus, 2-aminoquinoline (2-AQ) was isolated. This compound exhibited a modest MIC in vitro against strains from nine different BCC species, including multi-drug resistant clinical isolates (MIC $=8$ $64 \mu \mathrm{g} / \mathrm{ml})$, and a weak MIC $(128 \mu \mathrm{g} / \mathrm{ml})$ against $A$ baumannii. The $I C_{50}$ against a murine monocyte line was $1.5 \mathrm{mg} /$ $\mathrm{ml}$.

Conclusion: The small number of positive hits in this study suggests that finding a new drug from mushrooms to treat Gram-negative bacterial infections may be difficult. Although 2-AQ was identified in one mushroom, and it was shown to inhibit the growth of multi-drug resistant BCC isolates, the relatively high MICs $(8-128 \mu \mathrm{g} / \mathrm{ml})$ for both $A$. baumannii and BCC strains suggests that 2-AQ is not suitable for further drug development in its current form.

\section{Background}

Glucose nonfermenting, Gram-negative rods (GNFGNR) are commonly found in the environment and are typically responsible for opportunistic infections in severely ill and immunocompromised patients. Because this group of bacteria can survive in diverse

\footnotetext{
* Correspondence: schwan.will@uwlax.edu
'Department of Microbiology, University of Wisconsin-La Crosse, La Crosse,

* Correspondence: schwan.will@uwlax.edu
'Department of Microbiology, University of Wisconsin-La Crosse, La Crosse, WI, USA
}

(c) 2010 Schwan et al; licensee BioMed Central Ltd. This is an Open Access article distributed under the terms of the Creative Commons

environments, they are important opportunistic pathogens [1,2]. Pseudomonas aeruginosa is a leading cause of nosocomial infections due to GNF-GNR, followed by Acinetobacter spp., Stenotrophomonas maltophilia, and Alcaligenes spp. [3]. Although species in the Burkholderia cepacia complex (BCC) do not often cause human infections, it has been known for thirty years that infections that do occur are difficult to treat [4]. The BCC comprises a group of at least 17 species [5,6], although 
B. cenocepacia and B. multivorans are the most common species recovered from cystic fibrosis (CF) patients $[4,7]$. Besides CF patients, BCC also causes respiratory infections in hospitalized immunocompromised patients $[8,9]$. Some strains of the BCC are resistant to all known antibiotics, including the front line drugs, trimethoprim/ sulfamethoxazole, piperacillin, ceftazidime, ciprofloxacin, and pipericillin-tazobactam [10,11].

For the GNF-GNR, multidrug resistance is becoming increasingly common [3,12]. This has led to the loss of most classes of antibiotics to treat infections caused by this group of bacteria, resulting in the use of polymyxins as the only remaining option to treat many of the infections [12]. Unfortunately, polymyxins used for systemic infections carry with them an increased risk of nephrotoxicity in the patient population being treated. Moreover, BCC strains are inherently resistant to polymyxins.

Small molecules synthesized randomly through combinatorial chemistry techniques have been suggested as one possible solution to bacterial drug resistance; however, these molecules generally lack the complexity of those derived from natural sources $[13,14]$. Historically, broad screening of natural products for antibacterial activity has yielded many active compounds, with several being derived from secondary metabolites of the mycelium of filamentous fungi [15]. In addition, because of their natural resistance to degradation by many bacteria in the soil, fungal fruiting bodies (i.e. mushrooms) remain an untapped reservoir of novel natural products. Moreover, it is estimated that only $5 \%$ of the approximately 1.5 million fungal species have been described and chemically characterized [16]. Thus, wild mushrooms represent a potentially rich, untapped source of new antimicrobial agents. In this study, we screened a library of crude extracts from mushrooms for activity against $A$. baumannii and B. cepacia, hoping to find a new natural drug that could be used to treat infections caused by multi-drug resistant strains of these species.

\section{Methods}

\section{Construction of a mushroom crude extract library}

Three hundred and thirty wild mushrooms were collected from across the United States. Most the mushrooms were collected in Minnesota and Wisconsin, although some were found in Colorado, Illinois, Michigan, New Jersey, North Carolina, Oregon, Pennsylvania, and Tennessee. A total of 59 different mushroom families were represented in this collection. The family Tricholomataceae was represented most often (26 mushrooms) followed by the family Agaricaceae (16 mushrooms). Mushroom sample weights from each site ranged from $10 \mathrm{~g}$ to $70 \mathrm{~g}$, representing one to 25 fruiting bodies. Each mushroom was identified to the species level based on the structural characteristics of the fruiting body and air-dried in a commercially available food dehydrator. Five grams of each dried fungal material was extracted for $4 \mathrm{~h}$ at room temperature in the dark with $20 \mathrm{~mL}$ of a 5\% methanol-95\% dichloromethane solution. After filtration, the volatiles were removed by evaporation under a stream of $\mathrm{N}_{2}$ gas to yield the crude extract in a tared glass vial.

\section{Strains}

The bacterial strains used in this study are listed in Table 1. All Burkholderia strains were identified to the species level at the Burkholderia cepacia Reference Laboratory and Repository (University of Michigan) by polyphasic analyses using phenotypic and genotypic assays as described previously [8]. All Burkholderia isolates also were subjected to repetitive extragenic element-PCR typing using the BOX A1R primer as previously described [17] to ensure that all isolates were genotypically distinct. Bacteria were stored at $-80^{\circ} \mathrm{C}$ in skim milk or Luria-Bertani broth (Invitrogen) with 15\% glycerol and recovered from frozen stock overnight at $37^{\circ} \mathrm{C}$ on Mueller-Hinton (MH; Difco) agar. Bacteria were grown on trypticase soy agar (BBL) overnight at $35^{\circ} \mathrm{C}$ before use in susceptibility testing.

\section{Antimicrobial agents}

The following antibiotics were used in Kirby Bauer disk diffusion and MIC assays: tetracycline, trimethoprimsulfamethoxazole, tobramycin, gentamicin, amikacin, aztreonam, ceftazidime, meropenem, imipenem, ticaricillin/clavulonic acid, piperacillin/tazobactam, ciprofloxacin, piperacillin, cefepime, and levofloxacin. The antibiotics were purchased from Fluka or Sigma-Aldrich. Disk diffusion assays

All Kirby Bauer disk diffusion assays were performed according to Clinical and Laboratory Standards Institute (CLSI) guidelines [18]. Tetracycline and dimethylsulfoxide (DMSO) were used as controls in these assays. A zone of inhibition of growth greater than $6 \mathrm{~mm}$ was considered positive in this screening procedure.

\section{Bioassay-guided separation}

For those extracts that showed a zone of inhibition of growth of either $A$. baumannii UWL or B. cepacia ATCC 25416, the extract was fractionated by successive thin-layer chromatography (TLC), using $500 \mathrm{~mm}$ thickness Whatman $20 \mathrm{~cm} \times 20 \mathrm{~cm}, 60 \AA$ silica gel plates with fluorescent indicator, eluting initially with $5 \%$ methanol-95\% dichloromethane and then 50\% isopropanol-50\% acetonitrile. Following development of the TLC plates, one fraction with activity against these bacteria was visualized under short wave UV light $(254 \mathrm{~nm})$ as a relatively low $R_{\mathrm{f}}$, violet-colored band following development of the TLC plates. Final purification of this bioactive compound was accomplished by preparative scale high performance liquid chromatography (HPLC) using a Waters HPLC system with diode array detector, 
Table 1 Bacteria used in this study

\begin{tabular}{|c|c|c|}
\hline Bacteria & Strain & Source/reference ${ }^{a}$ \\
\hline Achromobacter xylosoxidans & $M C$ & $M C$ \\
\hline Achromobacter xylosoxidans & ATCC 9220 & ATCC \\
\hline Acinetobacter baumannii & UWL & UWL \\
\hline Acinetobacter baumannii & ATCC 19606 & ATCC \\
\hline Burkholderia ambifaria & AU4587 & $B C R L R$ \\
\hline Burkholderia ambifaria & AU5203 & $B C R L R$ \\
\hline Burkholderia ambifaria & AU11161 & $B C R L R$ \\
\hline Burkholderia cepacia & ATCC 25416 & ATCC \\
\hline Burkholderia cepacia & AU0108 & $B C R L R$ \\
\hline Burkholderia cepacia & AU2720 & $B C R L R$ \\
\hline Burkholderia cepacia & AU2769 & BCRLR \\
\hline Burkholderia cepacia & AU7554 & BCRLR \\
\hline Burkholderia cenocepacia & $J 2315$ & $B C R L R$ \\
\hline Burkholderia cenocepacia & AU6550 & BCRLR \\
\hline Burkholderia cenocepacia & AU9292 & $B C R L R$ \\
\hline Burkholderia cenocepacia & AU10321 & $B C R L R$ \\
\hline Burkholderia dolosa & AU0794 & BCRLR \\
\hline Burkholderia dolosa & AU3271 & $B C R L R$ \\
\hline Burkholderia dolosa & AU9336 & $B C R L R$ \\
\hline Burkholderia dolosa & AU9628 & $B c R L R$ \\
\hline Burkholderia dolosa & AU12872 & $B C R L R$ \\
\hline Burkholderia gladioli & AU3431 & $B C R L R$ \\
\hline Burkholderia gladioli & AU4123 & $B C R L R$ \\
\hline Burkholderia gladioli & AU9405 & $B C R L R$ \\
\hline Burkholderia multivorans & ATCC 27616 & ATCC \\
\hline Burkholderia multivorans & AU5573 & $B C R L R$ \\
\hline Burkholderia multivorans & AU7455 & $B C R L R$ \\
\hline Burkholderia multivorans & AU10398 & $B C R L R$ \\
\hline Burkholderia multivorans & $A \cup 4507$ & $B C R L R$ \\
\hline Burkholderia pyroccinia & AU5468 & $B C R L R$ \\
\hline Burkholderia pyroccinia & AU7314 & $B C R L R$ \\
\hline Burkholderia pyroccinia & AU1114 & $B C R L R$ \\
\hline Burkholderia stabilis & AU4757 & $B C R L R$ \\
\hline Burkholderia stabilis & AU5832 & BCRLR \\
\hline Burkholderia stabilis & AU9035 & $B C R L R$ \\
\hline Burkholderia vietnamiensis & AU3032 & BCRLR \\
\hline Burkholderia vietnamiensis & AU3578 & BCRLR \\
\hline Burkholderia vietnamiensis & AU3997 & $B C R L R$ \\
\hline Burkholderia vietnamiensis & AU5003 & $B C R L R$ \\
\hline Burkholderia vietnamiensis & AU10214 & $B C R L R$ \\
\hline Pseudomonas aeruginosa & ATCC 27853 & ATCC \\
\hline Stenotrophomonas maltophilia & UWL & UWL \\
\hline Stenotrophomonas maltophilia & ATCC 13637 & ATCC \\
\hline
\end{tabular}

${ }^{a}$ ATCC, American Type Culture Collection; BcRLR, Burkholderia cepacia Research Laboratory and Repository (University of Michigan);UWL, the University of Wisconsin-La Crosse Culture Collection; MC, Marshfield Clinic. reverse phase C-18 column (Alltech), and a 1\% ammonia-55\% methanol-44\% water solution as the eluant. The major component that eluted from the column retained the previously observed bioactivity, and this purified sample also was used for subsequent structure elucidation studies. Structural analyses were performed using positive ionization high-resolution mass spectrometry (HRMS; Varian Saturn mass spectrophotometer), proton and carbon NMR spectroscopy (500 MHz), and IR spectroscopy (thin film method).

MIC assays

All MIC assays were performed according to CLSI regulations [18]. B. cepacia ATCC 25416 was used as a control for these assays. Each strain was tested a minimum of three separate times to ensure reproducibility. Commercially available 2-AQ (Sigma-Aldrich) was used in side-by-side comparison testing with the naturally derived 2-AQ and in the screening of several BCC strains.

\section{Cytotoxicity assays}

To screen for cytotoxicity, two assays were used. The first assay used sheep blood agar plates and two-fold dilutions of the compound in phosphate buffered saline (PBS, pH 7.2). Eight microliters of the diluted drug were added to a $6 \mathrm{~mm}$ paper disk on a sheep blood agar plate. The plates were incubated for 16 to $18 \mathrm{~h}$ at $35^{\circ} \mathrm{C}$. A zone of lysis was considered positive and the last dilution exhibiting a hemolysis zone was considered the $\mathrm{IC}_{50}$ for the drug.

The second assay utilized was a trypan exclusion assay [19]. Murine J774A.1 (ATCC) and human U937 (ATCC) monocyte cell lines were tested. Monocytes were seeded at $1 \times 10^{6} /$ well in a 24 -well tissue culture plate with RPMI 1640 medium (Invitrogen). The next day, two-fold dilutions of the drug in PBS were added to each well with RPMI 1640 medium. On the following day, the spent medium was removed by aspiration and a trypan blue solution was added to each well for $10 \mathrm{~min}$. After the 10 min elapsed, the trypan blue solution was aspirated and an equal volume of PBS was added. Three fields of cells were counted per well. The number of dead (blue) and live (clear) cells was determined. An $\mathrm{IC}_{50}$ was calculated for the well with $50 \%$ dead cells.

\section{Results and Discussion}

Screening the mushroom library

New drugs are needed to treat infections caused by Gram-negative bacteria. The Antimicrobial Availability 
Task Force (AATF) of the Infectious Diseases Society of America (IDSA) has listed several microorganisms that are viewed as significant health problems [20]. Among the species listed are the GNF-GNR species $P$. aeruginosa and A. baumannii, predominantly because of the antibiotic resistance profiles for both species and the dearth of potentially effective drugs in the late-stage development pipeline. Moreover, extensively drug resistant $A$. baumannii that are even resistant to colistin are associated with high mortality rates [21]. In general, the pipeline for viable/strong candidate antibacterial drugs is lacking [22-24]. Currently, there are no new drugs with novel mechanisms of action in Phase I clinical studies to treat Gram-negative bacterial infections [25]. Furthermore, there are very few preclinical leads that exhibit favorable profiles against Gram-negative bacteria.

In an attempt to find new drugs to treat infections caused by GNF-GNR, 330 wild mushroom crude extracts were tested for their activity against $A$. baumannii UWL and B. cepacia ATCC 25416, but only three extracts showed a zone of inhibition of growth greater than $6 \mathrm{~mm}$ against both strains. The DMSO controls displayed no zone of inhibition (reported as 6 $\mathrm{mm}$ ), whereas tetracycline displayed a large inhibition zone against both species. In the subsequent screening of the three crude extracts, an extract from the Leucopaxillus albissimus mushroom was identified as the only one showing promising activity against either $A$. baumannii UWL or B. cepacia ATCC 25416 The zone of antibacterial activity of this extract was larger against $B$. cepacia compared to the zone for A. baumannii.

Purification of the active compound from an L. albissimus crude extract

The major active component from L. albissimus was determined to be 2-aminoquinoline (2-AQ), also known as 2-quinolylamine, on the basis of its spectral and physical data. Its positive ionization high-resolution mass spectrum (HRMS) showed an exact mass of 145.07573 $\mathrm{g} / \mathrm{mol}$ for the $\mathrm{M}+1$ ion, indicating a molecular formula of $\mathrm{C}_{9} \mathrm{H}_{8} \mathrm{~N}_{2}$ consistent with that of $2-\mathrm{AQ}$. The proton and carbon NMR spectra were essentially identical to those reported in the literature for 2-AQ [26]. The IR spectrum (thin film method) of the natural product was superimposable on the IR spectrum for 2-AQ contained in the online SDBS spectral database. Finally, the HPLC retention time and NMR spectra of the purified natural product were compared with those of an authentic synthetic sample obtained through Sigma-Aldrich, and these were identical, as were the biological activities of the two samples.

Antibacterial activity assessment of 2-AQ

Although antibacterial activity was previously attributed to 2-AQ [26,27], GNF-GNR species, such as BCC, $P$. aeruginosa, A. xylosoxidans, A. baumannii, and $S$. maltophilia, were not tested in earlier studies. To determine the in vitro activity of the 2-AQ natural product, a $\mathrm{MIC}$ analysis was performed to quantitatively measure its activity against BCC species and other GNF-GNR [13]. The MIC of 2-AQ for B. cepacia ATCC 25416 was $32 \mu \mathrm{g} / \mathrm{ml}$, but it was $16 \mu \mathrm{g} / \mathrm{ml}$ for B. cenocepacia J2315 and B. multivorans ATCC 27616. The MIC of 2-AQ was $64 \mu \mathrm{g} / \mathrm{ml}$ for S. maltophilia ATCC 13637, $32 \mu \mathrm{g} / \mathrm{ml}$ for A. xylosoxidans ATCC 9220, and $128 \mu \mathrm{g} / \mathrm{ml}$ for both P. aeruginosa ATCC 27853 and A. baumannii ATCC 19607 (TABLE 2). No inhibitory activity of 2-AQ was observed for Escherichia coli, Staphylococcus aureus, or Enterococcus faecalis (data not shown). Commercially available 2-AQ was also tested side-by-side with the natural product against the same species. No MIC difference was observed comparing the natural product to the commercially prepared sample. The same bacterial strains were tested against tetracycline, a drug class that has been proven effective against a wide variety of GNFGNR bacteria, and the results are also shown in TABLE 2. Although tetracycline was more active against some of the bacteria tested, the tetracycline MIC was equivalent against B. cepacia ATCC $25416(32 \mu \mathrm{g} / \mathrm{ml})$, two- to four-fold higher against the two A. xylosoxidans strains $(128 \mu \mathrm{g} / \mathrm{ml})$, and four-fold higher against $B$. cenocepacia $\mathrm{J} 2315$ when compared to the 2 -AQ MIC $(64 \mathrm{mg} / \mathrm{ml})$. In addition, the MICs of piperacillin and ceftazidime against the ATCC strains of B. cepacia and P. aeruginosa were both $1 \mu \mathrm{g} / \mathrm{ml}$. Moreover, the MIC of sulfamethoxazole-trimethoprim (SXT) against B. cepacia ATCC 25416 was $0.5 \mu \mathrm{g} / \mathrm{ml}$.

To determine if 2-AQ had broad anti-Burkholderia activity, a diverse panel of BCC strains as well as several Burkholderia gladioli strains were tested using the commercially prepared 2-AQ. These strains represented $B$.

Table 2 MICs of 2-aminoquinoline (2-AQ) isolated from $L$. albissimus and tetracycline (Tc) against several bacterial species

\begin{tabular}{llrr}
\hline Species & Strain & \multicolumn{1}{c}{ MIC $^{\mathbf{a}}$} \\
\hline & & 2-AQ & Tc $^{\mathbf{b}}$ \\
Pseudomonas aeruginosa & ATCC 27853 & 128 & 8 \\
Burkholderia cepacia & ATCC 25416 & 32 & 32 \\
Burkholderia cenocepacia & J2315 & 16 & 64 \\
Burkholderia multivorans & ATCC 27616 & 16 & 2 \\
Stenotrophomonas maltophilia & UWL & 32 & 0.25 \\
Stenotrophomonas maltophilia & ATCC 13637 & 64 & 1 \\
Achromobacter xylosoxidans & MC & 64 & 128 \\
Achromobacter xylosoxidans & ATCC & 32 & 128 \\
Acinetobacter baumannii & UWL & 128 & 0.5 \\
Acinetobacter baumannii & ATCC 19606 & 128 & 2 \\
\hline
\end{tabular}

${ }^{a}$ MIC given in $\mu \mathrm{g} / \mathrm{ml}$.

b $2-A Q, 2$-aminoquinoline; TC, tetracycline. 
gladioli and eight species within the BCC. Some of the strains were environmental isolates, whereas others were multi-drug and pan-drug resistant strains collected from hospitalized patients. These results showed that there was in vitro activity against $B$. gladioli and all of the BCC strains (MICs of 16 to $64 \mu \mathrm{g} / \mathrm{ml}$ ) (TABLE 3). Of the species assayed, the five $B$. cepacia strains had the lowest MIC range $(8-32 \mu \mathrm{g} / \mathrm{ml})$, whereas the $B$. stabilis had the highest MICs $(64 \mathrm{mg} / \mathrm{ml})$. Multi-drug resistant strains of B. ambifaria (AU5203 and AU11161), B. cepacia (AU0108), B. cenocepacia (all strains except J2313), B. dolosa (AU0936 and AU12872), B. multivorans (AU4507, AU5573, AU7455, and AU10398), B. pyroccinia (AU7324 and AU1114), B. stabilis (AU9035) and B. vietnamiensis (AU10214) were examined in this study. They all had 2-AQ susceptibility patterns similar to non-multi-drug resistant environmental isolates. Although B. gladioli is not part of the BCC, the strains that were tested also had high MICs $(64 \mathrm{mg} / \mathrm{ml})$. All of the $B$. cenocepacia clinical isolates tested either displayed full resistance to all antibacterial drugs (AU10321) or intermediate and full resistance (AU9292 and AU6550) to the same panel of 13 drugs that included trimethoprim/sulfamethoxazole, tobramycin, gentamicin, amikacin, aztreonam, piperacillin, piperacillin/tazobactam, ticaricillin/clavulonic acid, ceftazidime, cefepime, meropenem, imipenem, and levofloxacin (TABLE 4) [28]. The in vitro activity of 2-AQ suggests that the molecule has weak activity against bacterial pathogens that affect CF patients, including highly drug resistant strains of $B$. cepacia complex.

In vitro cytotoxicity testing of 2-AQ

A crude cytotoxicity test using sheep red blood cells demonstrated that cell lysis occurred when the concentration of 2 -AQ was $\geq 1.6 \mathrm{mg} / \mathrm{ml}$, suggesting that the drug might have a low cytotoxic effect. Furthermore, trypan blue exclusion experiments using 2-AQ against J774A.1 and U937 murine monocytic cell lines demonstrated that it took $1.5 \mathrm{mg} / \mathrm{ml}$ of 2 -AQ to injure $50 \%$ of the eukaryotic cells and $500 \mu \mathrm{g} / \mathrm{ml}$ to injure either cell
Table 3 MIC range of commercially available 2-AQ activities against Burkholderia strains

\begin{tabular}{llc}
\hline Species $(\boldsymbol{\mu g} / \mathbf{m l})$ & Strains Tested & MIC Range \\
\hline B. ambifaria & 3 & $16-64$ \\
B. cenocepacia & 4 & $16-64$ \\
B. cepacia & 5 & $8-32$ \\
B. dolosa & 5 & $32-64$ \\
B. gladioli & 3 & 64 \\
B. multivorans & 5 & 32 \\
B. pyroccinia & 3 & $32-64$ \\
B. stabilis & 3 & 64 \\
B. vietnamiensis & 5 & $32-64$ \\
\hline
\end{tabular}

line. These results demonstrate that 2-AQ has a cytotoxicity profile against mammalian cells only 10 to 30 fold higher than its MICs against GNF-GNR.

In this study, 2-AQ was identified as a natural product that targets GNF-GNR, particularly the BCC. With the number of multi-drug resistant $\mathrm{BCC}$ strains on the rise $[12,28,29]$, a new drug is sorely needed, particularly to treat BCC strains that display resistance to the frontline antibacterial drugs currently in use $[29,30]$. The in vitro MIC values for 2-AQ were the lowest against the $B$. cepacia strains within the $\mathrm{BCC}$; however, they were still too high to be considered for further commercial development of 2-AQ in its current form. Furthermore, 2AQ is commercially available and is considered to be a toxic compound. It is possible that an analog of $2-\mathrm{AQ}$ could be developed with a more favorable therapeutic index. Presently, 2-AQ analogs are being developed as melanin-concentrating hormone antagonists [31,32].

\section{Conclusion}

Screening of a library of wild mushroom extracts for antibacterial activity against $A$. baumannii and B. cepacia yielded limited results. Only three mushroom extracts displayed any activity, and further investigation of the mushroom with the most promising activity, $L$. albissimus, resulted in the isolation of a known

Table 4 Comparison of commercially prepared 2-AQ MICs versus frontline drug MICs against multidrug-resistant $B$. cepacia complex clinical isolates

\begin{tabular}{|c|c|c|c|c|c|c|c|c|c|c|c|c|c|c|}
\hline \multirow[b]{2}{*}{ Bacterial strain } & \multirow[b]{2}{*}{$\mathrm{SXT}^{\mathrm{a}}$} & \multirow[b]{2}{*}{ NN } & \multirow[b]{2}{*}{ GM } & \multirow[b]{2}{*}{ AN } & \multicolumn{10}{|c|}{ Antibacterial drug MIC ( $\mu \mathrm{g} / \mathrm{ml})$} \\
\hline & & & & & ATM & PIP & TZP & TIC & CAZ & FEP & MEM & IPM & LVX & 2-AQ \\
\hline B. multivorans AU5573 & 0.25 & 256 & 64 & 64 & 16 & 32 & 8 & 128 & 8 & 8 & 4 & 32 & 1 & 64 \\
\hline B. multivorans AU7455 & 1 & 64 & $>64$ & $>64$ & 16 & 8 & 8 & 32 & 8 & 2 & 2 & 32 & 2 & 32 \\
\hline B. multivorans AU10398 & 1 & $>256$ & $>64$ & $>64$ & 32 & $-b$ & 32 & $>128$ & $>64$ & $>32$ & 8 & 16 & 16 & 16 \\
\hline B. cenocepacia AU6550 & 4 & $>256$ & $>64$ & $>64$ & $>64$ & - & 128 & $>128$ & 16 & $>32$ & 16 & $>32$ & 16 & 16 \\
\hline B. cenocepacia AU9292 & $>4$ & $>256$ & $>64$ & $>64$ & $>64$ & $>128$ & $>128$ & $>128$ & 32 & $>32$ & 16 & $>32$ & $>16$ & 64 \\
\hline B. cenocepacia AU10321 & 4 & $>256$ & $>64$ & $>64$ & $>64$ & $>64$ & $>128$ & $>128$ & 64 & $>32$ & 32 & $>32$ & $>16$ & 32 \\
\hline
\end{tabular}

${ }^{a} \mathrm{SXT}=$ trimethoprim/sulfa, NN = tobramycin, GM = gentamicin, AN = amikacin, ATM = aztreonam, PIP = piperacillin, TZP = piperacillin/tazobactam, TIC = ticaricillin/clavulanic acid,, CAZ = ceftazidime, FEP = cefepime, MEM = meropenem, IPM = imipenem, LVX = levofloxacin, and 2-AQ = 2-aminoquinoline. b $-=$ not determined. 
compound, 2-AQ, that had modest activity against several BCC strains and limited potential for commercial development.

\section{Acknowledgements}

We wish to thank Mary Stemper from Marshfield Clinic for the $A$. xylosoxidans strain. This study was funded by a University of Wisconsin System Applied Research Grant to WRS. JJL receives funding from the Cystic Fibrosis Foundation.

\section{Author details}

'Department of Microbiology, University of Wisconsin-La Crosse, La Crosse, WI, USA. ${ }^{2}$ Department of Chemistry, University of Wisconsin-La Crosse, La Crosse, WI, USA. ${ }^{3}$ Department of Biology, University of Wisconsin-La Crosse, La Crosse, WI, USA. ${ }^{4}$ Department of Pediatrics, University of Michigan Medical School, Ann Arbor, MI, USA.

\section{Authors' contributions}

WRS performed laboratory studies, drafted the manuscript, and edited the manuscript; $\mathrm{AM}, \mathrm{MR}, \mathrm{KE}$, and TV created the mushroom library and edited the manuscript; $M G, K E, C D$, and TK screened the mushroom library and assisted with the purification of the active drug; JT performed the HPLC separation of the purified 2-AQ compound; JJL provided the clinical isolates, ran the MICs on the clinical isolates, and edited the manuscript; and RM and XTL obtained and interpreted the MS, IR, and NMR spectra. All authors read and approved the final manuscript.

\section{Competing interests}

WRS, AM, MR, and TV have filed a patent on 2-AQ.

\section{Received: 10 August 2009}

Accepted: 21 January 2010 Published: 21 January 2010

\section{References}

1. Bergogne-Berezin E, Towner KJ: Acinetobacter spp. as nosocomial pathogens: microbiological, clinical, and epidemiological features. Clin Microbiol Rev 1996, 9:148-165.

2. Lyczak JB, Cannon CL, Pier GB: Lung infections associated with cystic fibrosis. Clin Microbiol Rev 2002, 15:194-222.

3. Quinn JP: Clinical problems posed by multiresistant nonfermenting gram-negative pathogens. Clin Infect Dis 1998, 27(Suppl 1):S117-S124.

4. Tablan OC, Chorba TL, Schidlow DV, White JW, Hardy KA, Gilligan PH, Morgan WM, Carson LA, Martone WJ, Jason JM, Jarvis WR: Pseudomonas cepacia colonization in patients with cystic fibrosis: risk factors and clinical outcome. J Pediatr 1985, 107:382-387.

5. Vanlaere E, LiPuma JJ, Baldwin A, Henry D, Brandt ED, Mahenthiralingham E, Speert D, Dowson C, Vandamme P: Burkholderia latens sp. nov., Burkholderia diffusa sp. nov., Burkholderia arboris sp. nov., Burkholderia seminalis sp. nov., and Burkholderia metallica sp. nov.; five novel species within the Burkholderia cepacia complex. Int I Syst Evol Microbiol 2008, 58:1580-1590

6. Vanlaere E, Baldwin A, Gevers D, Henry D, De Brandt E, LiPuma JJ, Mahenthiralingam E, Speert DP, Dowson C, Vandamme P: Taxon $\mathrm{K}$ complex within the Burkholderia cepacia complex, comprises at least two novel species, Burkholderia contaminans sp. nov. and Burkholderia lata sp. nov. Int J Syst Evol Microbiol 2009, 59:102-111.

7. Coenye T, Vandamme O, Govan JR, LiPuma JJ: Taxonomy and identification of the Burkholderia cepacia complex. I Clin Microbiol 2001, 39:3427-3436.

8. Reik R, Spilker T, LiPuma JJ: Distribution of Burkholderia cepacia complex species among isolates recovered from persons with or without cystic fibrosis. J Clin Microbiol 2005, 43:2926-2928.

9. Holmes A, Nolan R, Taylor R, Finley R, Riley M, Jiang RZ, Steinbach S, Goldstein R: An epidemic of Burkholderia cepacia transmitted between patients with and without cystic fibrosis. J Infect Dis 1999, 179:1197-205.

10. Chen JS, Witzmann KA, Spilker T, Fink RJ, LiPuma JJ: Endemicity and intercity spread of Burkholderia cepacia genomovar III in cystic fibrosis. J Pediatr 2001, 139:643-649.
11. Golini G, Cazzola G, Fontana R: Molecular epidemiology and antibiotic susceptibility of Burkholderia cepacia -complex isolates from an Italian cystic fibrosis centre. Eur J Clin Microbiol Infect Dis 2006, 25:175-180.

12. McGowan JE Jr: Resistance in nonfermenting gram-negative bacteria: multidrug resistance to the maximum. Am J Infect Control 2006, 34(Suppl 1):S29-S37.

13. Feher M, Schmidt JM: Property distributions: differences between drugs, natural products, and molecules from combinatorial chemistry. J Chem Infect Comput Sci 2003, 43:218-227.

14. Shu Y-Z: Recent natural products based development: A pharmaceutical industry perspective. J Nat Prod 1998, 61:1053-1071.

15. Demain AL: Pharmaceutically active secondary metabolites of microorganisms. Appl Microbiol Biotechnol 1999, 52:455-463.

16. Hawksworth DL: The magnitude of fungal diversity: the 1.5 million species estimate revisited. Mycol Res 2001, 105:1422-1432.

17. Coenye T, LiPuma JJ: Multilocus restriction typing: A novel tool for studying global epidemiology of Burkholderia cepacia. complex infection in cystic fibrosis. J Infect Dis 2002, 185:1454-1462.

18. Clinical and Laboratory Standards Institute: Performance standards for antimicrobial susceptibility testing, $16^{\text {th }}$ informational supplement. NCCLS document M100-S16. National Committee for Clinical Laboratory Standards, Wayne, PA 2006.

19. Hathaway WE, Newby LA, Githens JH: The acridine orange viability test applied to bone marrow cells. I. Correlation with trypan blue and eosin dye exclusion and tissue culture transformation. Blood 1964, 23:517-525.

20. Talbot GH, Bradley J, Edwards JE Jr, Gilbert D, Scheld M, Bartlett JG: Bad bugs need drugs: an update on the development pipeline from the Antimicrobial Availability Task Force of the Infectious Diseases Society of America. Clin Infect Dis 2006, 42:657-668.

21. Doi Y, Husain S, Potoski BA, McCurry KR, Paterson DL: Extensively drugresistant Acinetobacter baumannii. Emerg Infect Dis 2009, 15:980-982.

22. Bush $\mathrm{K}$ : Why it is important to continue antibacterial drug discovery. ASM News 2004, 70:282-287.

23. DiMasi JA: New drug development in the United States from 1963 to 1999. Clin Pharmacol Ther 2001, 69:286-296.

24. Shlaes DM, Projan SJ, Edwards JE: Antibiotic discovery: state of the state. ASM News 2004, 70:275-281.

25. Payne DJ, Gwynn MN, Holmes DJ, Pompliano DL: Drugs for bad bugs: confronting the challenges of antibacterial discovery. Nat Rev 2007, 6:29-40.

26. Pfister JR: Isolation and bioactivity of 2-aminoquinoline from Leucopaxillus albissimus. J Nat Prod 1988, 51:969-970.

27. Arai I, Nakayama I: Antibacterial properties of pyridine and quinoline-Noxide derivatives. J Pharm Soc Jpn 1952, 72:167-172.

28. LiPuma JJ, Rathinavelu S, Foster BK, Keoleian JC, Makidon P, Kalikin LM, Baker JR Jr: In vitro activity of a novel nanoemulsion against Burkholderia and other multi-drug resistant cystic fibrosis-associated bacterial species. Antimicrob Agents Chemother 2009, 53:249-255.

29. Mahenthiralingam E, Baldwin A, Dowson CG: Burkholderia cepacia complex bacteria: opportunistic pathogens with important natural biology. J App/ Microbiol 2008, 104:1539-1551.

30. Sader HS, Jones RN: Antimicrobial susceptibility of uncommonly isolated non-enteric gram-negative bacilli. Int J Antimicrob Agents 2005, 25:95-109.

31. Jiang J, Hoang $M$, Young JR, Chaung $D$, Eid $R$, Turner $C$, Lin $P$, Tong $X$ Wang J, Tan C, Feighner S, Palyha O, Hreniuk DL, Pan J, Sailer AW, MacNeil DJ, Howard A, Shearman L, Stribling S, Camacho R, Strack A, Ploeg Van der LH, Goulet MT, DeVita RJ: 2-Aminoquinoline melaninconcentrating hormone (MCH)1R antagonists. Bioorg Med Chem Lett 2006, 16:5270-5274

32. Ulven T, Little PB, Receveur JM, Frimurer TM, Rist O, Nørregaard PK, Högberg T: 6-Acylamino-2-amino-4-methylquinolines as potent melaninconcentrating hormone 1 receptor antagonists: structure-activity exploration of eastern and western parts. Bioorg Med Chem Lett 2006, 16:1070-1075.

doi:10.1186/1476-0711-9-4

Cite this article as: Schwan et al: Screening a mushroom extract library for activity against Acinetobacter baumannii and Burkholderia cepacia and the identification of a compound with anti-Burkholderia activity. Annals of Clinical Microbiology and Antimicrobials 2010 9:4. 